\title{
Fluorinated mannosides inhibit cellular fucosylation.
}

Johan F.A. Pijnenborg ${ }^{[a], \dagger}$, Emiel Rossing ${ }^{[a], \dagger}$, Marek Noga ${ }^{[b]}$, Willem Titulaer ${ }^{[a]}$, Raisa Veizaj ${ }^{[c]}$, Dirk J.

Lefeber $^{[b, c]}$ and Thomas J. Boltje ${ }^{\star[a]}$

[a] J.F.A. Pijnenborg, E. Rossing, W. Titulaer, Dr. T.J. Boltje

Department of Synthetic Organic Chemistry

Institute for Molecules and Materials, Radboud University

Heyendaalseweg 135, 6525AJ, Nijmegen, The Netherlands

t.boltje@science.ru.nl

[b] Dr. M. Noga, Prof. D.J. Lefeber

Department of Laboratory Medicine, Translational Metabolic Laboratory

Radboud Institute for Molecular Life Sciences, Radboud University Medical Center

Geert Grooteplein Zuid 10,6525GA, Nijmegen, The Netherlands

[c] R. Veizaj, Prof. D.J. Lefeber

Department of Neurology

Donders Institute for Brain, Cognition and Behavior, Radboud University Medical Center

Geert Grooteplein Zuid 10,6525GA, Nijmegen, The Netherlands

[†] These authors contributed equally to this work.

Supporting information for this article is given via a link at the end of the document.

Abstract: Fucose sugars are expressed on mammalian cell membranes as part of glycoconjugates and mediates essential physiological processes. The aberrant expression of fucosylated glycans has been linked to pathologies such as cancer, inflammation, infection, and genetic disorders. Tools to modulate fucose expression on living cells are needed to elucidate the biological role of fucose sugars and the development of potential therapeutics. Herein, we report a novel class of fucosylation inhibitors directly targeting de novo GDP-fucose biosynthesis. We demonstrate that cell permeable fluorinated mannoside 1-phosphate derivatives (Fucotrim I \& II) are metabolic prodrugs that are metabolized to their respective GDPmannose derivatives and efficiently inhibit cellular fucosylation.

a

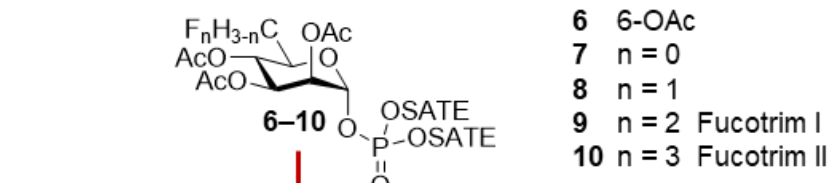

L-Fucose (Fuc) is a 6-deoxyhexose expressed at the termini of glycan chains that decorate cell surface proteins and lipids. ${ }^{1}$ The fucose residues on glycoconjugates are essential mediators of physiological processes. For example, the fucose moiety in the tetrasaccharide sialyl Lewis ${ }^{x}\left(s \mathrm{~L}^{\mathrm{x}}\right.$ ) expressed on leukocytes is recognized by selectin receptors that regulate leukocyte recruitment and extravasation. The aberrant expression of $\mathrm{sLe}^{\mathrm{x}}$ and increased fucosylation has been linked to pathologies, most notably cancer, and has been shown to promote tumor progression. ${ }^{2,3}$ Reducing fucosylation of glycans in cancer using inhibitors of the fucose biosynthesis has therefore been recognized as a promising therapeutic option. Glycans or proteins are fucosylated by the action of thirteen fucosyltransferases (FucTs) that differ in acceptor glycan preference but all utilize GDP-fucose as the donor substrate. ${ }^{4}$

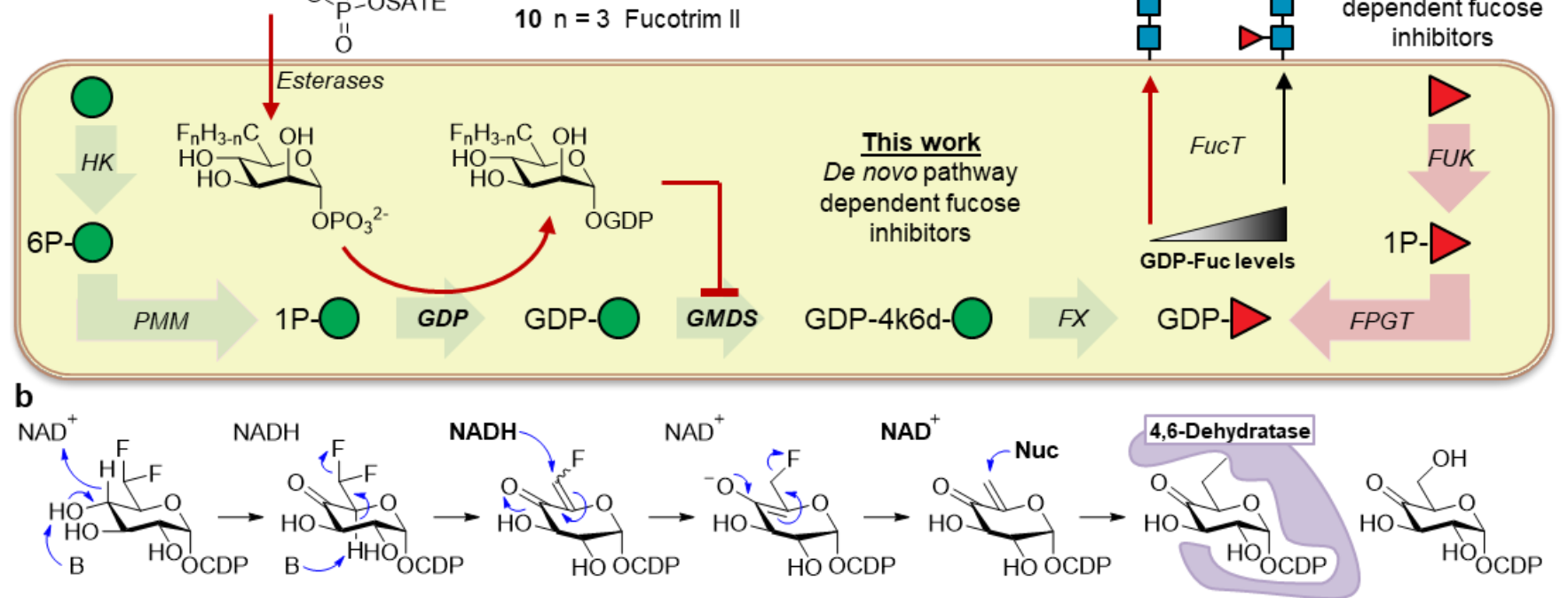

Scheme 1. (a) Working model of metabolic fucosylation inhibitors. (b) Previous work showing mechanism of action of different 4,6-dehydratase inhibitor acting on CDP-D-glucose 4,6-dehydratase. Abbreviations: HK, hexokinase; PMM, phosphomannomutase; GDP, mannose-1-phosphate guanylyltransferase; GMDS, GDPmannose 4,6 dehydratase; FX, GDP-4-keto-6-deoxy-D-mannose-3,5-epimerase-4-reductase (GMER); FUK, fucokinase; FPGT, fucose-1-phosphate guanylyltransferase. 
Cells generate GDP-fucose either via the recycling of fucose released during glycan turnover in the lysosome (salvage pathway) or via de novo biosynthesis from mannose 1-phosphate (Scheme 1). To decrease cellular fucosylation, fucosyltransferase (FucT) inhibitors have attracted considerable attention. $^{2}$ A common strategy has been mimicking the natural substrate guanosine diphosphate fucose (GDP-Fuc). However, the essential GDP moiety in these compounds is associated with a high polarity and low stability thereby limiting their use in vivo by poor penetration of the cell membrane. This hurdle was recently overcome with the development of less polar, cell permeable fucose derivatives which are metabolized to the corresponding active GDP-fucose analogs through the salvage pathway. $5,6,7,8$ These compounds target FucTs by competitive inhibition and the de novo enzymes GMDS and FX by feedback inhibition. This strategy led to fucosylation inhibitors that are active in vitro and in vivo, showing promising anticancer effects in liver, breast and blood cancer models. $9,10,11,12,13$ Moreover, combining a fucosylation inhibitor with immunotherapy vaccination in immunocompetent mice completely protected against tumor growth due to enhanced antibody-dependent cellular cytotoxicity (ADCC) with LS174T colorectal carcinoma and A20 lymphoma cells. ${ }^{11}$ These inhibitors enter via the fucose salvage pathway yet it is estimated that $\sim 90 \%$ of the GDP-Fuc pool is biosynthesized via de novo biosynthesis from GDP-mannose. ${ }^{14}$ Thus, direct inhibition of de novo GDP-fucose biosynthesis could result in more potent inhibitors yet this approach remains unexplored so far to the best of our knowledge. GDP-fucose is biosynthesized from GDP-mannose by oxidation of the 4-carbon to a ketone and dehydration of the 6-position by GDP-mannose-4,6-dehydratase (GMDS, Figure 3a). Subsequent epimerization of the 3- and 5position followed by the reduction of the 4-ketone by FX leads to GDP-Fucose. Hence, GMDS and FX are prime targets to develop de novo pathway inhibitors.

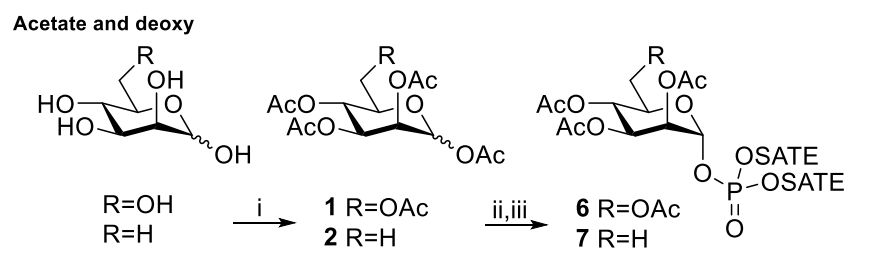

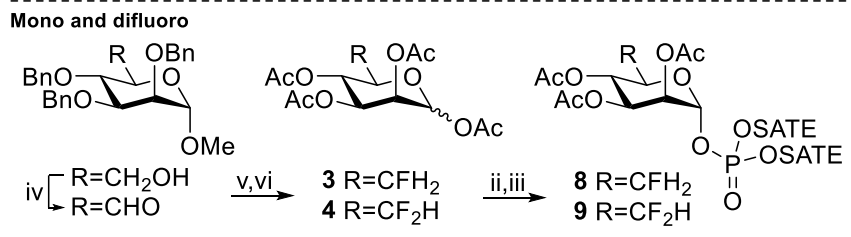

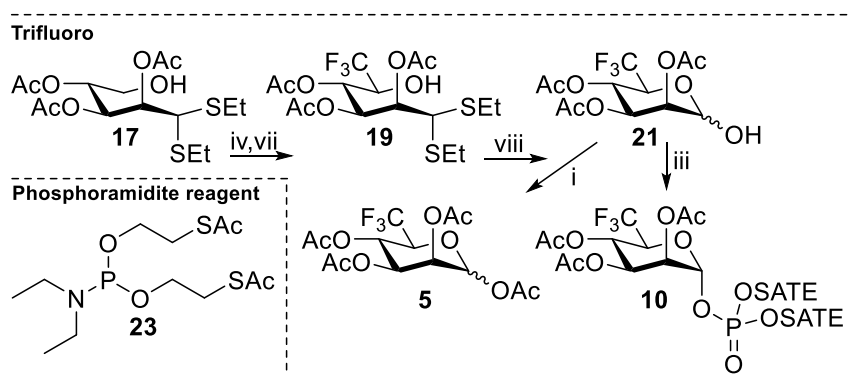

Scheme 2. Synthesis of 1-10. i) $\mathrm{Ac}_{2} \mathrm{O}$, $\mathrm{Pyr}$; ii) $\mathrm{H}_{2} \mathrm{NNH}_{2} \cdot \mathrm{HOAc}, \mathrm{DMF}$; iii) bis(Sacetyl-2-thioethyl) N,N-diethylphosphoramidite (23), $1 \mathrm{H}$-tetrazole, ACN, then mCPBA; iv) DMP, $\mathrm{NaHCO}_{3}, \mathrm{DCM}$; v) DAST, DCM; vi) $\mathrm{Ac}_{2} \mathrm{O}, \mathrm{H}_{2} \mathrm{SO}_{4}, \mathrm{AcOH}$; vii) $\mathrm{TMSCF}_{3}$, TBAF, THF, then $\mathrm{AcOH}$; viii) NBS, $\mathrm{H}_{2} \mathrm{O}$ /acetone.

Herein we report the development of highly potent inhibitors of the de novo pathway based on fluorinated mannose 1phosphate derivatives (Scheme 1a). ${ }^{15}$ We were inspired by a mechanism-based inhibitor for CDP-D-glucose 4,6-dehydratase isolated from Yersinia pseudotuberculosis. Use of a 6-deoxydifluoride modified CDP-glucose formed a reactive Michael acceptor inside the active site leading to entrapment (Scheme 1b)..$^{16}$ We hypothesized that GDP-D-6-deoxy-difluoro-mannose (GDP-D-Rha6F 2 ) could inhibit GMDS, also a 4,6-dehydratase, through a similar mechanism. To allow passive diffusion over the cell membrane, lipophilic metabolic precursors were designed. Two entry points in the de novo biosynthesis were considered with precursors based on mannose (1-5) and mannose-1-phosphate (6-10). The lipophilic (thio)ester protecting groups are expected to be cleaved by esterases inside the cell, allowing the derivatives to enter the endogenous GDP-mannose biosynthetic pathway. To investigate the degree of fluorination on the inhibitory potency we modified the 6-position with none to three fluorides (2-5 \& 7-10).

Compounds 1-10 were synthesized as described in Scheme 2 and the supplementary information. Compounds 1 and 2 were obtained by acetylation of D-mannose and D-rhamnose, respectively. Mono- and difluorides 3 and $\mathbf{4}$ were obtained via DAST mediated fluorination of the $6-\mathrm{OH}$ or aldehyde, respectively, followed by acetolysis. The synthesis of protected 1-phosphate analogs 6-9 was achieved in a two-step sequence from their respective precursors $1-4$. Selective deprotection of the anomeric acetyl ester was achieved using hydrazinium acetate. The resulting lactol was reacted with phosphoramidite reagent 23 bearing two $S$-acetyl-2-thioethyl (SATE) groups and subsequently oxidized to corresponding phosphate triesters 6-9. Trifluorides 5 and 10 were prepared via a homologation approach by introducing a $\mathrm{CF}_{3}$-group at the $\mathrm{C}-5$ position of the pentose $\mathrm{D}$ lyxose. To this end, derivative $\mathbf{1 7}$ was oxidized and reacted with a trifluoromethyl anion affording a separable mixture of the desired 6-trifluoro D-rhamnose (19) and the byproduct L-gulose (20) derivative. Hydrolysis of the thioacetal with NBS and water resulted in ring closure and the resulting lactol was acetylated or phosphorylated to afford 5 and $\mathbf{1 0}$, respectively. This synthetic approach ensures pyranose formation.

Table 1. $\mathrm{EC}_{50}$ values in micromolar for fucose expression inhibition. ${ }^{[\mathrm{a}]}$

\begin{tabular}{|c|c|c|c|c|c|c|}
\hline & THP-1 & & Jurkat & & EL4 & \\
\hline Compound & AAL & $\mathrm{AOL}$ & AAL & $\mathrm{AOL}$ & AAL & $\mathrm{AOL}$ \\
\hline DMSO & $\mathrm{NI}$ & $\mathrm{NI}$ & $\mathrm{NI}$ & $\mathrm{NI}$ & $\mathrm{NI}$ & $\mathrm{NI}$ \\
\hline P-D-Man-1P (6) & $\mathrm{NI}$ & $\mathrm{NI}$ & $\mathrm{NI}$ & $\mathrm{NI}$ & $\mathrm{NI}$ & $\mathrm{NI}$ \\
\hline P-D-Rha-1P (7) & $\mathrm{NI}$ & 137 & $\mathrm{NI}$ & 153 & $\mathrm{NI}$ & $\mathrm{NI}$ \\
\hline P-D-Rha6F-1P (8) & 267 & 84 & 174 & 345 & $\mathrm{NI}$ & $\mathrm{NI}$ \\
\hline P-D-Rha6F $F_{2}-1 P(9)$ & 2.0 & 5.3 & 5.9 & 4.1 & 14 & 38 \\
\hline P-D-Rha6F $3-1 P(\mathbf{1 0})$ & 0.61 & 0.45 & 28 & 13 & $\mathrm{NI}$ & $\mathrm{NI}$ \\
\hline P-Fuc2F & 45 & 31 & 115 & 121 & $\mathrm{NI}$ & $\mathrm{NI}$ \\
\hline
\end{tabular}

[a] Three cell lines (THP-1, Jurkat, EL4) were cultured for 3 days with 0-512 $\mu \mathrm{M}$ of compound $\mathbf{6 - 1 0}$, P-Fuc2F or DMSO control. The cells were stained with two fucose specific lectins (AAL \& AOL) and analyzed by flow cytometry, presented as mean percentage lectin binding normalized to control $(n=3)$. N.I, no inhibition $\left(\mathrm{EC}_{50}>500 \mu \mathrm{M}\right)$. 

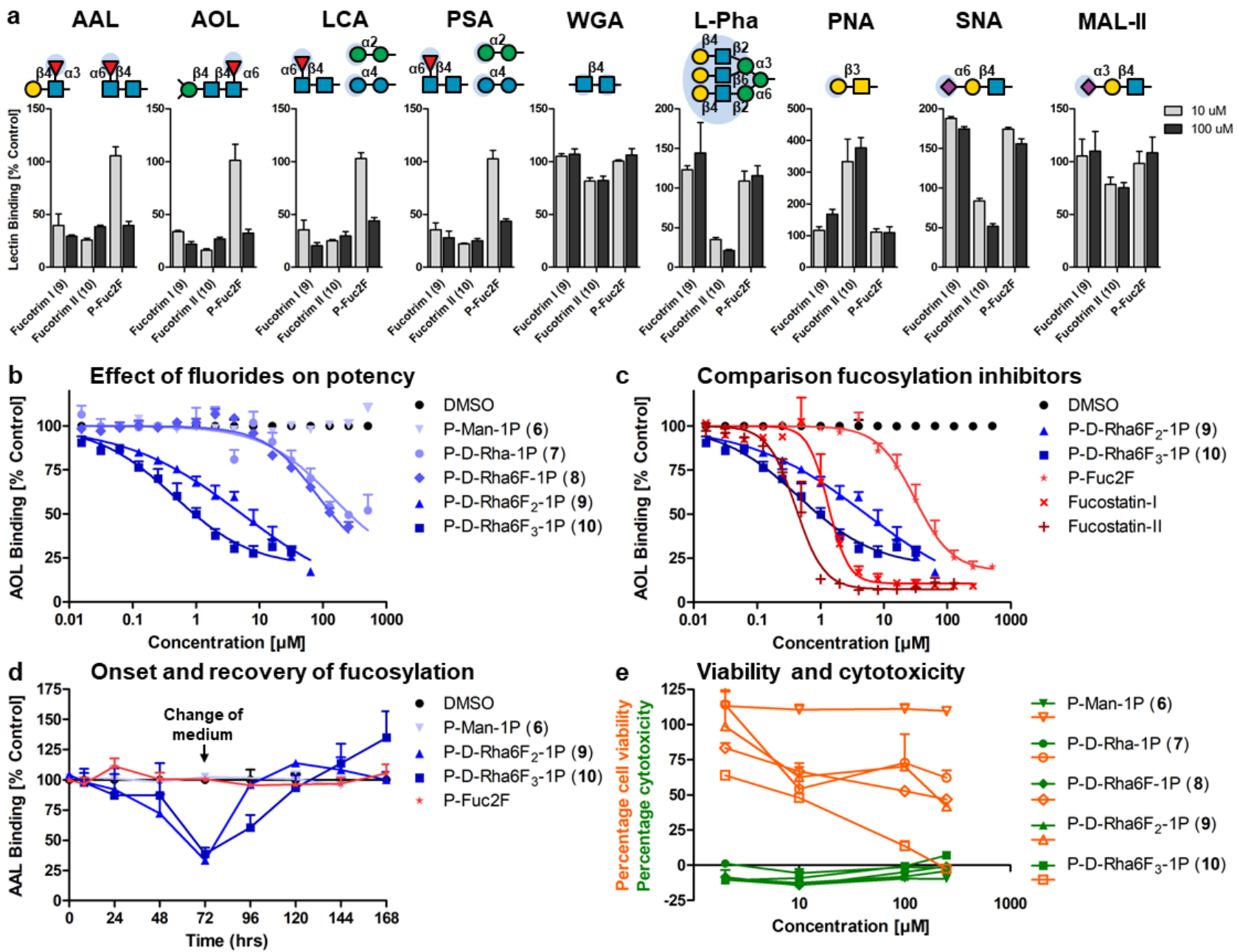

Figure 1. (a) Effect of 10 or $100 \mu \mathrm{M}$ Fucotrim I \& II and P-Fuc2F on cell surface glycosylation using different lectins, presented as percentage lectin binding compared to DMSO control. (b) Effect of C6-modifications on potency of inhibition. (c) Potency of Fucotrim I and II compared to known salvage pathway dependent fucose inhibitors P-Fuc2F, Fucostatin-I and Fucostatin-II using AOL lectin $(n=3)$. (d) Onset and recovery of defucosylation. THP1 cells were incubated with 10 or $100 \mu M$ (Figure S2) compound or DMSO control and fucosylation levels were determined with AAL lectins (Figure S2) for six days. (e) The effect on viability and cytotoxicity on THP1 cells after incubation with 2, 10, 100 or $250 \mu \mathrm{M}$ 6-10 or 1-5 (Figure S4) for three days was determined with an XTT and LDH respectively and presented as percentage cell viability or cytotoxicity compared to DMSO control.

The inhibitory potency of 1-10 was assessed at different concentrations in human THP-1 cells with fucose specific AAL and $A O L$ lectins (Figure S1a-d). The $E_{50}$ values were determined, defined as the concentration where a $50 \%$ decrease in lectin binding compared to control was observed (Table 1). The non-phosphorylated compounds 1-5 did not show any inhibition of fucosylation. In contrast, from the phosphorylated set $\mathbf{6 - 1 0}$, the mono-, di-, and trifluorinated derivatives 7-10 displayed inhibition of fucosylation. A trend was observed where increasing the number of C-6 fluorides increased its potency (Figure 1 $\mathbf{1 b}$ ). Inhibitors P-D-Rha6F $F_{2}-1 \mathrm{P}$ (Fucotrim I, 9) and P-D-Rha6F $\mathrm{F}_{3}-1 \mathrm{P}$ (Fucotrim II, 10) afforded (sub)micromolar inhibition of fucosylation. Both Fucotrim I (9) \& II (10) were more potent than fucosylation inhibitor P-Fuc2F (1,3,4-tri-O-acetyl-2-deoxy-2fluoro-L-fucose), a known salvage pathway dependent inhibitor. Also in human Jurkat cells potent inhibition for Fucotrim I and II was observed (Table 1; Figure S1e-f). In mouse cell line EL4 however, only Fucotrim I (9) inhibited fucosylation while both Fucotrim II (10) and P-Fuc2F did not inhibit fucosylation (Table 1; Figure S1g-h).
Since Fucotrim I and II (9\&10) are mannose-1-phosphate derivatives, it is possible that other glycosylation pathways such as mannosylation would be affected. Having established the ability to inhibit cellular fucosylation with $A A L$ and AOL lectins, the effect on total cell surface glycosylation was evaluated using additional lectins after 3 days incubation (Figure 1a; Figure S2). $\mathrm{N}$-glycans consist of a biantennary mannose containing backbone, recognized by WGA, and can be branched, recognized by L-Pha. These branches can be terminated with galactose, recognized by PNA, or $\alpha 2,6-$ and $\alpha 2,3-$ linked sialic acids recognized by SNA and MAL-II, respectively. ${ }^{17,18}$ Binding of LCA and PSA was decreased for all fucosylation inhibitors as they are core fucose dependent. The binding of WGA was unaffected as it recognizes core GIcNAc residues independent of fucosylation. ${ }^{17}$ Fucotrim I and P-Fuc2F showed a highly similar profile, not affecting the binding of L-Pha, PNA, and MAL-II. Only the binding of SNA increased using these inhibitors. Fucotrim II (10) on the other hand showed a less specific profile, decreasing the binding of L-Pha, SNA and MAL-II and increasing the binding of PNA. This would not be expected for a selective fucosylation inhibitor as sialylation is also clearly 
affected. From these results we conclude that Fucotrim I has a similar specificity as known fucosylation inhibitor P-Fuc2F but is more potent.

To further evaluate the potency of Fucotrim I and II, we made a direct comparison with known fucosylation inhibitors $P$ Fuc2F, P-L-Fuc6F 3 (Fucostatin I) and P-L-Fuc- $1 \mathrm{CH}_{2} \mathrm{P}$ (Fucostatin II) (Figure 1c). ${ }^{5,6,7}$ The $\mathrm{EC}_{50}$ values for known Fucostatin I and II were determined at $1.3 \mu \mathrm{M}$ and $0.41 \mu \mathrm{M}$ respectively, which is in the same range as Fucotrim I and II under these conditions (Table 1). P-Fuc2 $F$ was significantly less potent with an $E_{50}$ of $31 \mu \mathrm{M}$.

The onset of inhibition and duration for recovery was evaluated on THP-1 cells with 10 and $100 \mu \mathrm{M}$ compound using the AAL lectin (Figure 1d-e; Figure S3). In line with the potency data, no inhibition was observed for known inhibitor P-Fuc2F at $10 \mu \mathrm{M}$ concentration. At the same concentration, Fucotrim I and II decreased lectin binding by over $50 \%$ after 3 days with full recovery to normal fucosylation levels after 4-5 days. At $100 \mu \mathrm{M}$ concentration Fucotrim I and II inhibit fucosylation after 1 day and to over $50 \%$ after 3 days. Fucosylation was fully recovered to normal levels after 6 days. Similar results were obtained using the AOL lectin (Figure S3).

Finally, a toxicity profile of $\mathbf{1 - 1 0}$ was established by monitoring the metabolic activity and cell death of THP-1 cells after three days of treatment (Figure 1e; Figure S4). Importantly, none of the compounds were cytotoxic at concentrations up to $250 \mu \mathrm{M}$. However, the cell viability was decreased which might be attributed to the known relation between fucosylation and proliferation. ${ }^{9,10,19}$
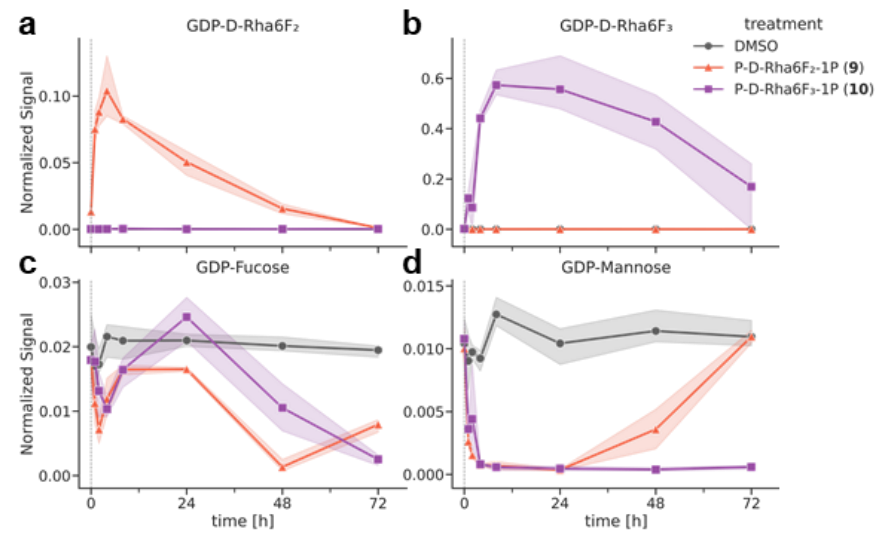

Figure 2. Nucleotide sugar analysis. THP-1 cells were incubated for indicated time points with $10 \mu \mathrm{M}$ Fucotrim I or II or with DMSO control. After sample preparation, the GDP-Rha6F 2 (a), GDP-Rha6F 3 (b), GDP-Fuc (c) and GDP-Man (d) levels were analyzed using reverse-phase ion pairing chromatography coupled to a triple quadrupole mass spectrometer operating in negative ion mode and presented as their abundance in the nucleotide sugar pool (Figure S5) $(n=3)$.

It has been suggested that the reduced proliferation after incubation with known fucosylation inhibitors is caused by acting on the de novo biosynthesis of GDP-fucose rather than acting on fucosyltransferases which correlates with our findings..$^{20}$

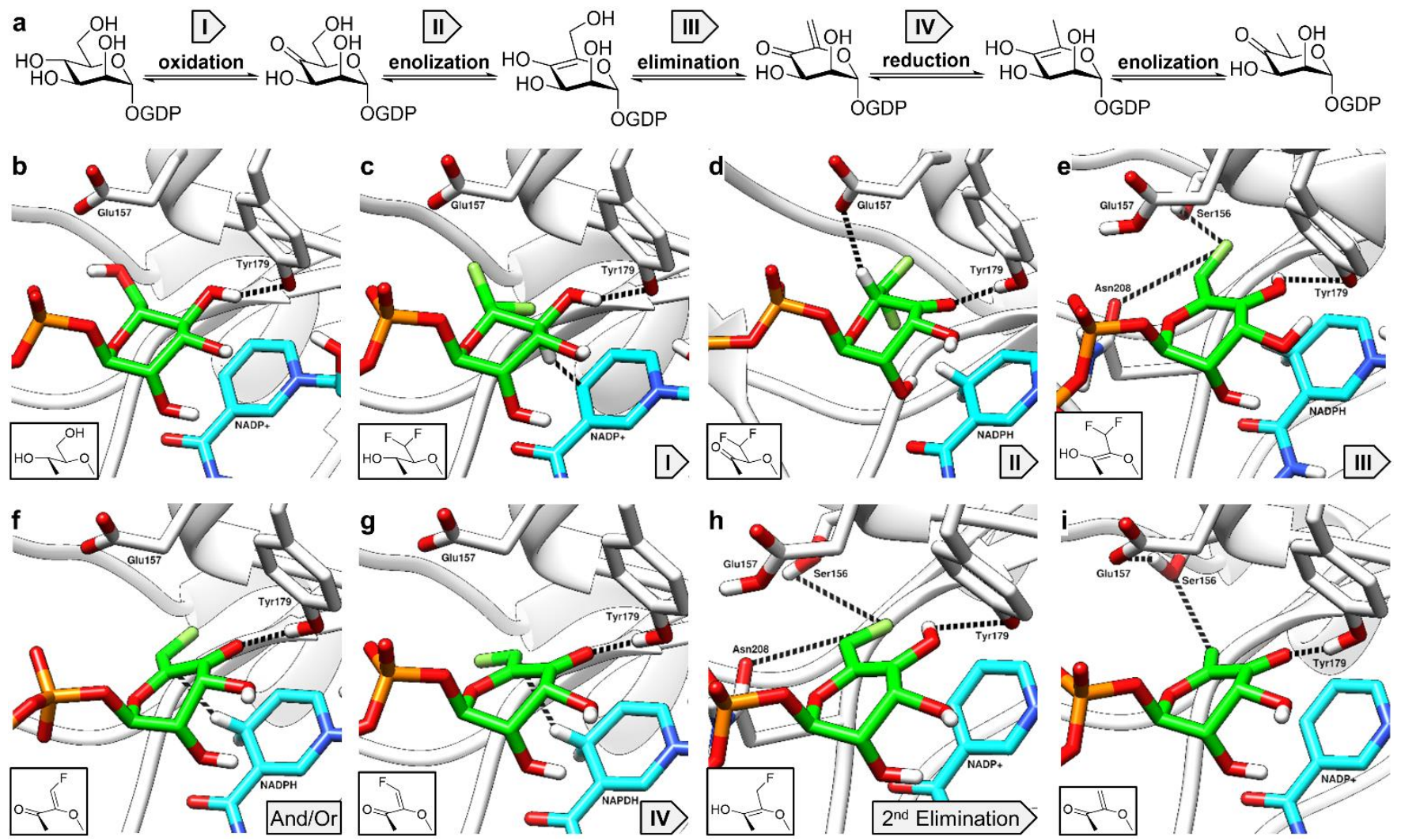

Figure 3. (a) Endogenous mechanism of action for human GMDS. ${ }^{21}$ (b) Natural substrate GDP-mannose (green carbons) was docked at the 4-fluoro-GDP-mannose (G4F) binding site in human GMDS (6GPJ ${ }^{21}$, white carbons) in which also NADP+ (cyan carbons) was cocrystalized. (c-i) Unnatural substrate GDP-D-Rha6F 2 (c, green carbons) and the proposed metabolic intermediates ( $d-i$, green carbons) were docked in the same active site. For each structure, the active site residues Glu157 and Tyr179 were first protonated or deprotonated and either NADPH or NADP+ was present accordingly with the known mechanism of action on GDP. mannose. Black dotted lines depict expected interactions between atoms leading to a new product, based on the natural mechanism. ${ }^{21}$ 
To test our hypothesized prodrug strategy, we studied the metabolism of Fucotrim I and II to their corresponding GDPanalogs inside cells. To this end, intracellular nucleotide sugar levels were analyzed. THP-1 cells were treated for different time points with Fucotrim I and II and extracted metabolites analyzed using reverse-phase ion pairing chromatography coupled to a triple quadrupole mass spectrometer operating in negative ion mode (Figure 2; Figure S5). For analysis of GDP-D-Rha6F 2 and GDP-D-Rha6F $_{3}$ analogs, theoretical mass transitions were programmed. After incubations for only 1 hour, both Fucotrim GDP-analogs were observed with a maximum abundance after 4-8 hours (Figure 2a). Subsequently, GDP-fucose levels decreased after 1-2 days for both inhibitors, indicating inhibition of GDP-fucose biosynthesis by Fucotrim I and II (Figure 2b). GDP-mannose levels were also affected by both inhibitors. For Fucotrim I, the GDP-mannose levels recovered after 3 days and for Fucotrim II, GDP-mannose levels didn't recover within the 3 days of the experiment. This correlates well with the lectin panel (Figure 1a) where after 3 days incubation Fucotrim I only shows an effect on fucosylation and Fucotrim II shows an effect on multiple forms of glycosylation. Importantly, for both Fucotrim I and II no changes were observed for other nucleotide sugar levels such as UDP-glucose and UDP-galactose (Figure S5).

Having established the metabolism towards GDP-D-Rha6 $\mathrm{F}_{2}$ and GDP-D-Rha6 $\mathrm{F}_{3}$ inside the cell we investigated if a mechanism of inhibition as previously described for a bacterial CDP-glucose 4,6-dehydratase (Figure 1b) would be plausible for Fucotrim I using in silico experiments. ${ }^{16}$ The docking experiments were based on a recent study describing the mechanism of action for human GMDS in detail (Figure 3a). ${ }^{21}$ The natural substrate GDPmannose was docked at the 4-fluoro-GDP-mannose (G4F) binding site in a human GMDS crystal structure (6GPJ) in which cofactor $\mathrm{NADP}^{+}$was co-crystallized (Figure $3 \mathbf{b}$ ). ${ }^{21}$ Docking of GDP-D-Rha6F $F_{2}$ in the same binding site resulted in similar poses as for GDP-mannose, suggesting it would still be a substrate for GMDS. The proposed metabolic intermediates (Figure $\mathbf{3 d - i )}$ ) are based on the endogenous mechanism of action for GMDS and a known 4,6-dehydratase inhibitor. ${ }^{16,21}$ The catalytic residues were positioned such that oxidation (I, Figure 3d), enolization (II, Figure $\mathbf{3 e}$ ), elimination (III, Figure $\mathbf{3} \mathbf{f}-\mathbf{g}$ ) and reduction (IV, Figure $3 \mathbf{h}$ ) do not seem to be affected sterically. In the endogenous mechanism enolization would yield the final product. For the difluoride derivative however, a second elimination might occur, affording an electrophilic intermediate (Figure 3i) that cannot be reduced due to the lack of reductant NADPH in the active site. It is hypothesized that this intermediate either competitively binds GMDS, or analogous to previous findings ${ }^{16}$, reacts with a nucleophilic amino acid (e.g. Ser156) in GMDS resulting in covalent inhibition, or with water affording a natural intermediate for GMDS. The in silico experiments indicate that GDP-D-Rha6F 2 likely acts on GMDS but further studies are needed to investigate the exact mechanism of action.

In conclusion, we have developed in vitro validated de novo pathway dependent fucosylation inhibitors. Fucotrim I (P-DRha6F $\left._{2}-1 \mathrm{P}, 9\right)$ and Fucotrim II (P-D-Rha6F $\left.\mathrm{F}_{3}-1 \mathrm{P}, 10\right)$ are new tools to study fucosylation function and are good candidates for further therapeutic development. Additionally, this approach could be considered for targeting other 4,6-dehydratases affording cell active inhibitors.

\section{Acknowledgements}

This work was supported by an ERC-Stg awarded to T.J.B., the Netherlands Organization for Scientific Research (VIDI Grant 91713359 to D.J.L), and the Radboud Consortium for Glycoscience. We thank prof. Gosse Adema PhD and the Molecular Immunology group at the Radiotherapy \& Oncolmmunology lab at the Radboud University Medical Centre for kindly supplying us with the THP-1, Jurkat and EL-4 cell lines. We would to acknowledge John Allen from Amgen Inc. for providing Fucostatin I and II.

Keywords: Fucosylation - Inhibition - De novo pathway • Small molecule $\cdot$ GMDS $\cdot$ mannoside

\section{References:}

1. Becker, D. J.; Lowe, J. B., Fucose: biosynthesis and biological function in mammals. Glycobiology 2003, 13 (7), 41R-53R.

2. Tu, Z.; Lin, Y.-N.; Lin, C.-H., Development of fucosyltransferase and fucosidase inhibitors. Chemical Society Reviews 2013, 42 (10), 4459-4475.

3. Varki, A., Essentials of Glycobiology. 3 ed.; 2017; pp 597-609.

4. Narimatsu, Y.; Joshi, H. J.; Nason, R.; Van Coillie, J.; Karlsson, R.; Sun, L.; Ye, Z.; Chen, Y.-H.; Schjoldager, K. T.; Steentoft, C.; Furukawa, S.; Bensing, B. A.; Sullam, P. M.; Thompson, A. J.; Paulson, J. C.; Büll, C.; Adema, G. J.; Mandel, U.; Hansen, L.; Bennett, E. P.; Varki, A.; Vakhrushev, S. Y.; Yang, Z.; Clausen, H., An Atlas of Human Glycosylation Pathways Enables Display of the Human Glycome by Gene Engineered Cells. Molecular Cell 2019, 75 (2), 394407.e5.

5. Rillahan, C. D.; Antonopoulos, A.; Lefort, C. T.; Sonon, R.; Azadi, P.; Ley, K.; Dell, A.; Haslam, S. M.; Paulson, J. C., Global metabolic inhibitors of sialyl- and fucosyltransferases remodel the glycome. Nat Chem Biol 2012, 8 (7), 661-668.

6. Kizuka, Y.; Nakano, M.; Yamaguchi, Y.; Nakajima, K.; Oka, R.; Sato, K.; Ren, C.-T.; Hsu, T.-L.; Wong, C.-H.; Taniguchi, N., An Alkynyl-Fucose Halts Hepatoma Cell Migration and Invasion by Inhibiting GDP-Fucose-Synthesizing Enzyme FX, TSTA3. Cell Chemical Biology 2017, 24 (12), 1467-1478.e5.

7. Allen, J. G.; Mujacic, M.; Frohn, M. J.; Pickrell, A. J.; Kodama, P.; Bagal, D.; San Miguel, T.; Sickmier, E. A.; Osgood, S.; Swietlow, A.; Li, V.; Jordan, J. B.; Kim, K.-W.; Rousseau, A.-M. C.; Kim, Y.-J.; Caille, S.; Achmatowicz, M.; Thiel, O.; Fotsch, C. H.; Reddy, P.; McCarter, J. D., Facile Modulation of Antibody Fucosylation with Small Molecule Fucostatin Inhibitors and Cocrystal Structure with GDP-Mannose 4,6-Dehydratase. ACS Chemical Biology 2016, 11 (10), 2734-2743.

8. Zandberg, W. F.; Kumarasamy, J.; Pinto, B. M.; Vocadlo, D. J., Metabolic inhibition of sialyl-Lewis $X$ biosynthesis by 5 -thiofucose remodels the cell surface and impairs selectin-mediated cell adhesion. J Biol Chem 2012, 287 (47), 40021-40030.

9. Zhou, Y.; Fukuda, T.; Hang, Q.; Hou, S.; Isaji, T.; Kameyama, A.; Gu, J., Inhibition of fucosylation by 2 -fluorofucose suppresses human liver cancer HepG2 cell proliferation and migration as well as tumor formation. Sci Rep 2017, 7 (1), 11563-11563.

10. Carrascal, M. A.; Silva, M.; Ramalho, J. S.; Pen, C.; Martins, M.; Pascoal, C.; Amaral, C.; Serrano, I.; Oliveira, M. J.; Sackstein, R. Videira, P. A., Inhibition of fucosylation in human invasive ductal carcinoma reduces E-selectin ligand expression, cell proliferation, and ERK1/2 and p38 MAPK activation. Mol Oncol 2018, 12 (5), 579593.

11. Okeley, N. M.; Alley, S. C.; Anderson, M. E.; Boursalian, T. E. Burke, P. J.; Emmerton, K. M.; Jeffrey, S. C.; Klussman, K.; Law, C. L.; Sussman, D.; Toki, B. E.; Westendorf, L.; Zeng, W.; Zhang, X.; Benjamin, D. R.; Senter, P. D., Development of orally active inhibitors of protein and cellular fucosylation. Proc Natl Acad Sci U S A 2013, 110 (14), 5404-5409. 
12. Okeley, N. M.; Heiser, R. A.; Zeng, W.; Hengel, S. M.; Wall, J.; Haughney, P. C.; Yap, T. A.; Robert, F.; Sanborn, R. E.; Burris, H.; Chow, L. Q.; Do, K. T.; Gutierrez, M.; Reckamp, K.; Weise, A.; Camidge, D. R.; Strickler, J.; Steuer, C.; Wang, Z.; O'Meara, M. M.; Alley, S. C.; Gardai, S. J., Abstract 5551: SGN-2FF: A small-molecule inhibitor of fucosylation modulates immune cell activity in preclinical models and demonstrates pharmacodynamic activity in early phase 1 analysis. Cancer Research 2018, 78 (13 Supplement), 5551-5551.

13. Disis, M. L.; Corulli, L. R.; Gad, E. A.; Koehnlein, M. R.; Cecil, D. L.; Senter, P. D.; Gardai, S. J.; Okeley, N. M., Therapeutic and prophylactic anti-tumor activity of an oral inhibitor of fucosylation in spontaneous mammary cancers. Molecular Cancer Therapeutics 2020, molcanther.0500.2019.

14. Yurchenco, P. D.; Atkinson, P. H., Fucosyl-glycoprotein and precursor pools in HeLa cells. Biochemistry 1975, 14 (14), 3107-3114. 15. Pijnenborg, J.F.A.; Rossing, E.; Boltje, T.J., New fluorinated hexoses. EP20195308.0, 2020

16. Chang, C.-W. T.; Chen, X. H.; Liu, H.-w., CDP-6-deoxy-6,6difluoro-d-glucose: A Mechanism-Based Inhibitor for CDP-d-glucose 4,6-Dehydratase. Journal of the American Chemical Society 1998, 120 (37), 9698-9699.

17. Gao, C.; Hanes, M. S.; Byrd-Leotis, L. A.; Wei, M.; Jia, N.; Kardish, R. J.; McKitrick, T. R.; Steinhauer, D. A.; Cummings, R. D., Unique Binding Specificities of Proteins toward Isomeric AsparagineLinked Glycans. Cell Chemical Biology 2019, 26 (4), 535-547.e4.

18. Iskratsch, T.; Braun, A.; Paschinger, K.; Wilson, I. B. H. Specificity analysis of lectins and antibodies using remodeled glycoproteins. Analytical Biochemistry 2009, 386 (2), 133-146.

19. Jia, L.; Zhang, J.; Ma, T.; Guo, Y.; Yu, Y.; Cui, J., The Function of Fucosylation in Progression of Lung Cancer. Front Oncol 2018, 8, 565-565.

20. Dai, Y.; Hartke, R.; Li, C.; Yang, Q.; Liu, J. O.; Wang, L.-X., Synthetic Fluorinated L-Fucose Analogs Inhibit Proliferation of Cancer Cells and Primary Endothelial Cells. ACS Chemical Biology 2020.

21. Pfeiffer, M.; Johansson, C.; Krojer, T.; Kavanagh, K. L.; Oppermann, U.; Nidetzky, B., A Parsimonious Mechanism of Sugar Dehydration by Human GDP-Mannose-4,6-dehydratase. ACS Catalysis 2019, 9 (4), 2962-2968. 


\section{Entry for the Table of Contents}

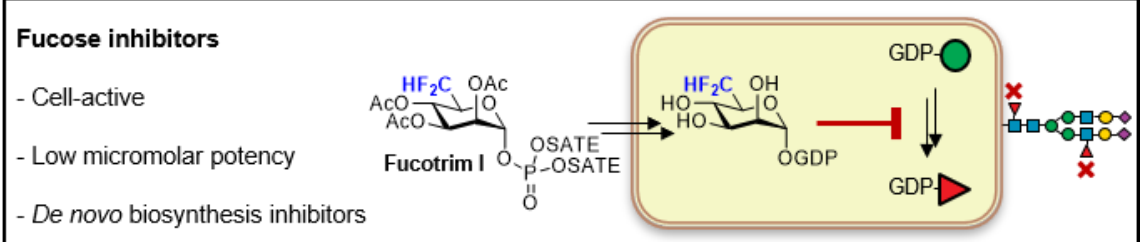

Fucose sugars on mammalian cell membranes play important roles in physiological and pathological processes. Inhibition of fucosylation has been identified as a therapeutic avenue. We have developed potent cell-active fucosylation inhibitors, directly inhibiting the de novo biosynthesis of GDP-fucose.

Institute and/or researcher Twitter usernames: @JohanPijnenborg 